\title{
A Spectral Analysis of the Central Component of CTB 80
}

\author{
G. Castelletti, G. Dubner \\ Instituto de Astronomía y Física del Espacio (CONICET, UBA), \\ C.C.67, Suc. 28, 1428 Buenos Aires, Argentina \\ K. Golap, W. M. Goss \\ National Radio Astronomy Observatory, P.O. Box 0, Socorro, New \\ Mexico 87801, USA
}

\begin{abstract}
Based on observations of the extended supernova remnant CTB 80 carried out with the Giant Metrewave Radio Telescope (GMRT, India) at 240 and $618 \mathrm{MHz}$ we have analyzed the spectral behavior of the $\sim 10^{\prime}$ nebula formed near the pulsar PSR B1951+32, finding signatures of the interaction of the pulsar with the surrounding plasma.
\end{abstract}

\section{Introduction}

The supernova remnant (SNR) CTB 80 (at a distance of $\sim 2 \mathrm{kpc}$, Strom \& Stappers 2000) presents three asymmetric arms (each about half a degree in size) with filamentary structure which overlap in a bright and elongated nebula about $10^{\prime} \times 6^{\prime}$ in size $\left(\alpha \sim-0.3\right.$, where $\left.S_{\nu} \propto \nu^{\alpha}\right)$. This nebula, named the "plateau" in the literature (Angerhofer et al. 1981), hosts in the western end a very bright compact nebula, called the "core", powered by the fast spinning pulsar PSR B1951+32. The core, about $1^{\prime}$ in size, is substantially polarized (Angerhofer et al. 1981) and has a spectral index $\alpha \sim 0.0$ (Strom 1987). PSR $\mathrm{B} 1951+32$, with a $\sim 40 \mathrm{~ms}$ pulse period, is moving toward the southwest with a relatively high velocity of $\sim 240 \mathrm{~km} \mathrm{~s}^{-1}$ (Migliazzo et al. 2002).

We have recently conducted a high-resolution study of the whole SNR CTB 80 at $240,330,618$ and $1380 \mathrm{MHz}$ using the VLA and GMRT radio interferometers (Castelletti et al. 2003). The GMRT image at $618 \mathrm{MHz}$ (beam $10^{\prime \prime} \times 6^{\prime \prime}$ ) has revealed that the plateau nebula is not smooth, but structured. Particularly, a prominent feature looking like a twisted filament, about $6^{\prime}$ along, appears to be trailing from the bright compact "core" formed around the pulsar.

\section{A Tomographic Analysis of the Pulsar Wind Nebula in CTB 80}

The radio images at 240 and $618 \mathrm{MHz}$ were regrided and convolved to a common angular resolution of $36^{\prime \prime} \times 23^{\prime \prime}$ at a P.A. $=67^{\circ}$. Based on these images, we have constructed a gallery of tomographic images to identify different spectral components in the central nebula (Katz-Stone, Rudnick \& Anderson 1993). The technique consists of the use of a test spectral index $\alpha_{t}$ as a probe to explore 

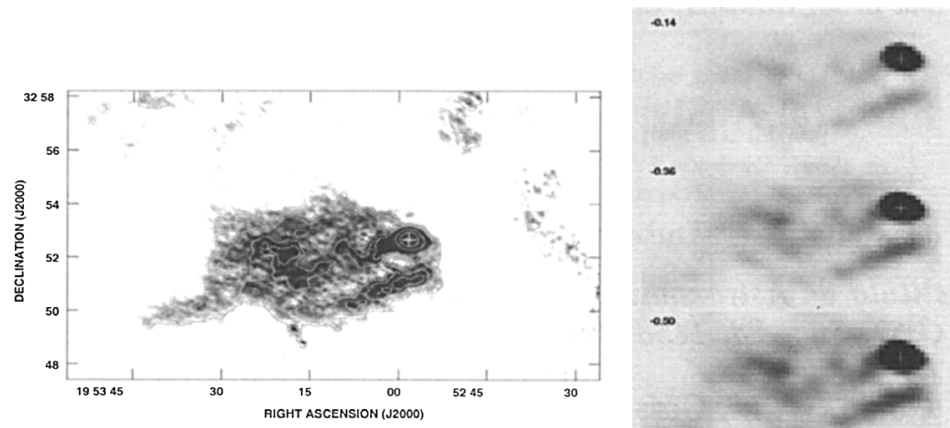

Figure 1. The plateau nebula at $618 \mathrm{MHz}$ (left) and three tomographic maps between 240 and $618 \mathrm{MHz}$ (right). The pulsar's position is indicated by a plus sign. The $\alpha_{t}$ values are shown at the top left corner of each tomographic map.

the spatial spectral distribution. The regions that have a spectrum flatter or steeper than $\alpha_{t}$ will appear darker or lighter than the gray background. In brief, for each map black features have a flatter spectral index than white features.

In Figure 1 we show the resulting tomographic images between 240 and 618 $\mathrm{MHz}$ for three test $\alpha_{t}=-0.14,-0.36$, and -0.50 . Apart from the compact core, with a spectral index close to 0.0, these images show that the helical filament observed in the plateau nebula as trailing from the pulsar, unequivocally has a spectrum flatter than the surroundings. A gradient in spectral index can also be observed across this feature, steepening from the core to the eastern extreme. We propose that this helical filament is a signature of the interaction of PSR B1951+32 with the surrounding plasma, either showing the path of the pulsar in its motion towards the west (the gradient would be caused by the aging of the particles), or illuminating the active injection of accelerated particles by the pulsar into the surrounding plasma. The origin of the flat spectrum observed in the long filament located to the southwest of the plateau nebula is not clear.

\section{References}

Angerhofer, P. E., Strom, R. G., Velusamy, T., \& Kundu, M. R. 1981, A\&A, 94, 313

Castelletti, G., Dubner, G., Golap, K., Goss, W. M., Velázquez, P. F., Holdaway, M., \& Rao, A. P. 2003, AJ, 126, 2114

Katz-Stone, D. M., Rudnick, L., \& Anderson, M. C. 1993, ApJ, 407, 549

Migliazzo, J. M., Gaensler, B. M., Backer, D. C., Stappers, B. W., van der Swaluw, E., \& Strom, R. G. 2002, ApJ, 567, L141

Strom, R. G. 1987, ApJ, 319, L103

Strom, R. G., \& Stappers, B. W. 2000, in ASP Conf. Ser., Vol. 202, Pulsar Astronomy - 2000 and Beyond, eds. M. Kramer, N. Wex, \& R. Wielebinski, (San Francisco: ASP), p. 509 